\title{
Commentary: Genomic Analysis Reveals Heterogeneity Between Lesions in Synchronous Primary Right-Sided and Left-Sided Colon Cancer
}

\begin{abstract}
José Perea ${ }^{1,2 *}$, Luis Corchete ${ }^{3}$, Juan L. García ${ }^{3}$, Miguel Urioste ${ }^{4}$ and Rogelio González-Sarmiento ${ }^{5}$

${ }^{1}$ Surgery Department, Fundación Jiménez Díaz University Hospital, Madrid, Spain, ${ }^{2}$ Fundación Jiménez Díaz Research Institute, Madrid, Spain, ${ }^{3}$ Hematology Department, Institute of Biomedical Research of Salamanca (IBSAL), Cancer Research Center (CiCIBMCC, CSIC/USAL), Center for Biomedical Research in Network of Cancer (CIBERONC), University Hospital of Salamanca, Salamanca, Spain, ${ }^{4}$ Familial Cancer Clinical Unit, Human Cancer Genetics Program, Spanish National Cancer Research Center (CNIO), Madrid, Spain, ${ }^{5}$ Molecular Medicine Unit, Biomedical Research Institute of Salamanca (IBSAL), Institute of Molecular and Cellular Biology of Cancer (IBMCC), University of Salamanca-USAL-CSIC, Salamanca, Spain
\end{abstract}

Keywords: synchronous colorectal cancer, clonality, metachronous colorectal cancer, heterogeneity, colorectal cancer

\section{OPEN ACCESS}

Edited by:

Chandan Kumar-Sinha,

University of Michigan, United States

Reviewed by:

José Manuel Lopes,

Universidade do Porto, Portugal

*Correspondence:

José Perea

josepereag@hotmail.com

Specialty section:

This article was submitted to

Molecular Diagnostics and

Therapeutics,

a section of the journa

Frontiers in Molecular Biosciences

Received: 28 October 2021

Accepted: 29 December 2021

Published: 21 January 2022

Citation:

Perea J, Corchete L, García JL, Urioste $M$ and González-Sarmiento $R$

(2022) Commentary: Genomic

Analysis Reveals Heterogeneity

Between Lesions in Synchronous

Primary Right-Sided and Left-Sided

Colon Cancer.

Front. Mol. Biosci. 8:803707.

doi: 10.3389/fmolb.2021.803707

\section{A Commentary on}

Genomic Analysis Reveals Heterogeneity Between Lesions in Synchronous Primary Right-Sided and Left-Sided Colon Cancer

by Hu H., Zhang Q., Huang R., Gao Z., Yuan Z., Tang Q., Gao F., Wang M., Zhang W., Ma T., Qiao T., Jin Y., Wang G. (2021). Front. Mol. Biosci. 8:689466. doi: 10.3389/fmolb.2021.689466

We read with interest the recent study by $\mathrm{Hu}$ et al., in which they described an approach to elucidate the genomic landscape of synchronous colorectal cancer (SCRC). They used a cohort of paired tumors located in different sites of the colon (right and left colon) and analyzed single nucleotide variation, somatic mutation, and copy number alteration by whole-exome sequencing. The authors finally suggested the heterogeneity between lesions and the polyclonal origin of the paired tumors in the same individual ( $\mathrm{Hu}$ et al., 2021). They added other previous studies also reporting the heterogeneity and independent genetic origin of SCRC (Cereda et al., 2016; Wang et al., 2018), but without focusing on the location of the paired tumors. On the contrary, to the best of our knowledge, we previously analyzed the largest series of SCRC in exploring clonality, composed by 104 paired SCRCs from 52 consecutive patients without hereditary forms of CRC, using initially a single-nucleotide polymorphism array and a subsequent statistical application to define them according to clonality. Moreover, we used parameters like the mutational concordance and $\mathrm{CpG}$ island methylator phenotype (CIMP) status, to confirm our clonality results, and developed a classification according to clonality and paired tumor location, showing clinical correlations. Our results suggested heterogeneity in $64.4 \%$ of cases (Figure 1A) but the presence of clonality in $35.6 \%$ of cases (Figure 1B) (Perea et al., 2019).

The importance of the method used to define clonality between paired tumors, multiple primary neoplasms, or metastatic disease, should also be critical. In this regard, most authors analyzed each case independently employing diverse approaches, such as focusing solely on shared mutations 
A

Patient 1 , odds in favor of independence $=2.1 \mathrm{e}+03$

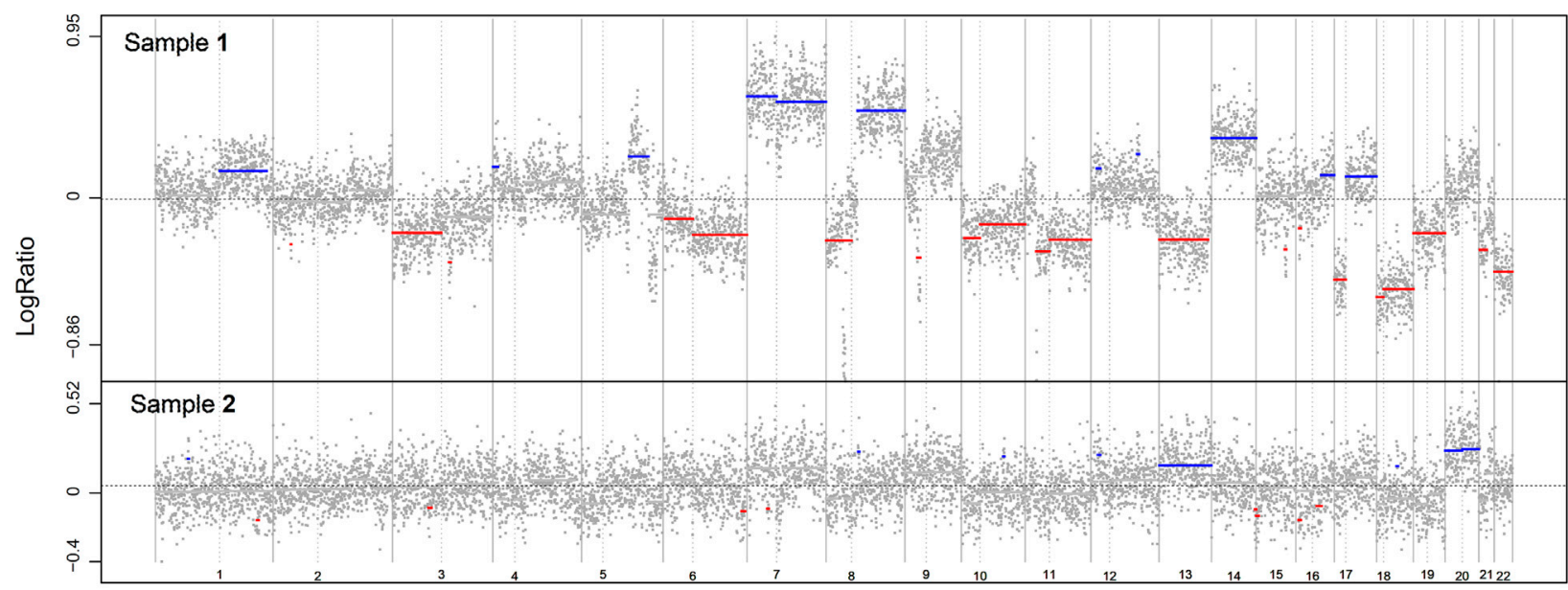

B

Patient 4 , odds in favor of clonality (metastasis) $=3.4 \mathrm{e}+05$

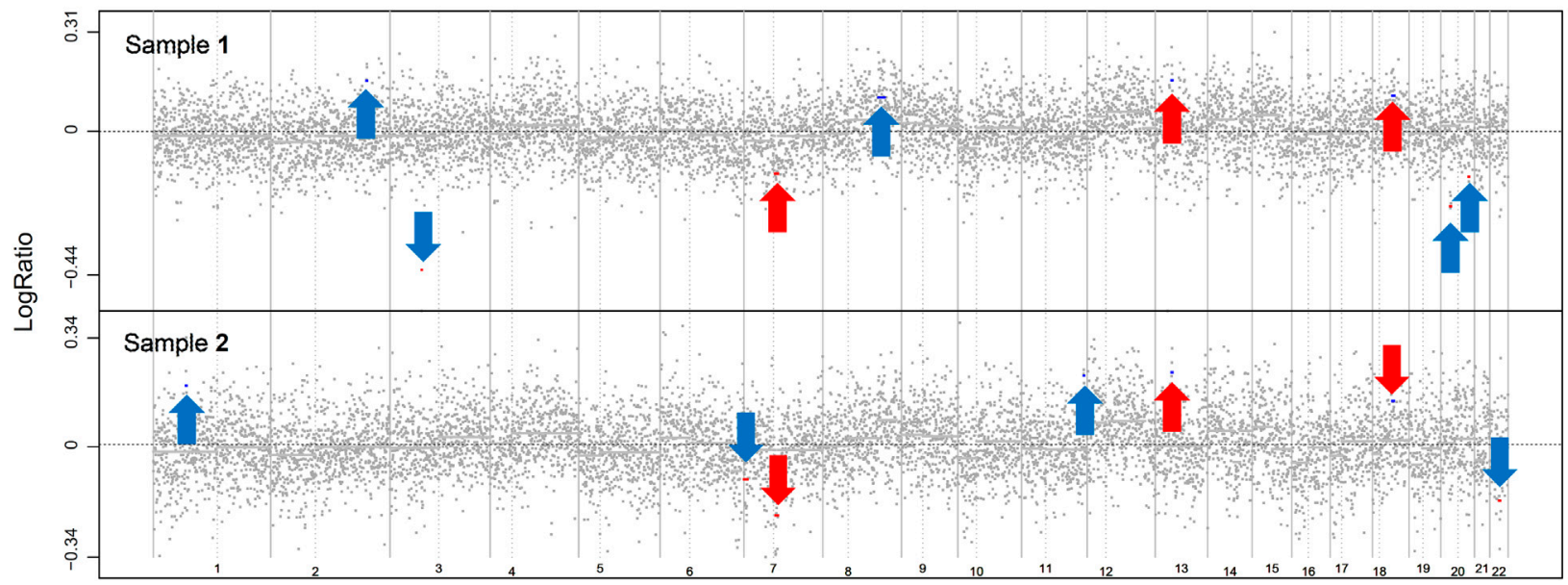

FIGURE 1 | Examples of the Clonality R package genome-wide results based on the likelihood ratio analysis (own data). (A) Example of two independent tumors from a CRC patient. (B) Example of two clonal tumors from a CRC patient. Red arrows indicate similar copy number (CN) segments. Blue arrows indicate clonal-specific $\mathrm{CN}$ segments. Gray dots represent the $\log _{2}$ ratios for each genomic position. Chromosome gains are depicted in blue and losses in red.

between paired tumors, ignoring the information from mutations that occur in one tumor but not the other, evidence that argues against clonal relatedness (Teixeira et al., 2004; Schultheis et al., 2015). Other authors have used the proportion of observed mutations that are shared as the index (Cereda et al., 2016), or assuming that the matched mutations follow a binomial distribution (Bao et al., 2015). Mauguen et al. analyzed the data from all cases collectively to obtain parametric estimates of the proportion of cases in the population that are clonal, and also count heavily on the recognition of the fact that the probabilities of occurrence of the observed mutations are crucially informative, especially for shared mutations (Mauguen et al., 2019). The comparison between the leftsided and right-sided lesions carried out by $\mathrm{Hu}$ et al. with SNP signature and tumor mutation burden was performed by a two-tailed paired $t$-test alone, while our results showing clonality were based on a likelihood ratio statistic calculated by the $\mathrm{R}$ software package Clonality (Ostrovnaya et al., 2011), which quantifies the odds that the two tumors are clonal. This method uses the copy number profiles from all samples, to determine whether two tumors from the same patient were clonal or origin-independent.

In our previous work (Perea et al., 2019), we classified the location of the paired tumors as monosegmental, when both were in the same colon segment (right or left colon), and pancolonic, when both were distributed all over the entire colon (right and left colon). The distribution of tumors according to the tumor location showed equivalent distribution for both monosegmental and pancolonic cases, with 65.5 and $61 \%$ of heterogeneous/polyclonal tumors, respectively. The use of a small cohort is also a limitation, also pointed out by $\mathrm{Hu}$ et al., and the need for larger studies with enough cases appears 
necessary to build a solid definition and approach to define clonal tumors, independently of the type of multiple paired tumors (multiple primary cases or metastasis and primary neoplasm).

Regarding other types of multiple primary colorectal cancers (CRCs), such as metachronous colorectal cancers, recent studies have hypothesized that a small proportion could also be from the same origin, as they showed similar molecular signatures (Backes et al., 2019). An analysis of metachronous CRCs (MCRCs) performed by Backes et al. led the authors to hypothesize that primary tumor cells might be seeded in a new location after biopsy of the primary tumor. In this study, only three cases were considered, and we think that the clonal theory should not be discarded. Taking into account both groups of clonal CRCs regarding both SCRCs and MCRCs, we think that, after subsequent prospective larger studies, the concept of SCRCs and MCRCs could be redefined. Therefore, those cases of

\section{REFERENCES}

Backes, Y., Seerden, T. C. J., van Gestel, R. S. F. E., Kranenburg, O., Ubink, I., Schiffelers, R. M., et al. (2019). Tumor Seeding during Colonoscopy as a Possible Cause for Metachronous Colorectal Cancer. Gastroenterology 157 (5), 1222-1232. doi:10.1053/j.gastro.2019.07.062

Bao, L., Messer, K., Schwab, R., Harismendy, O., Pu, M., Crain, B., et al. (2015). Mutational Profiling Can Establish Clonal or Independent Origin in Synchronous Bilateral Breast and Other Tumors. PLOS ONE 10 (11), e0142487. doi:10.1371/journal.pone.0142487

Cereda, M., Gambardella, G., Benedetti, L., Iannelli, F., Patel, D., Basso, G., et al. (2016). Patients with Genetically Heterogeneous Synchronous Colorectal Cancer Carry Rare Damaging Germline Mutations in Immune-Related Genes. Nat. Commun. 7, 12072. doi:10.1038/ncomms 12072

Hu, H., Zhang, Q., Huang, R., Gao, Z., Yuan, Z., Tang, Q., et al. (2021). Genomic Analysis Reveals Heterogeneity between Lesions in Synchronous Primary Right-Sided and Left-Sided Colon Cancer. Front. Mol. Biosci. 8 (8), 689466. doi:10.3389/fmolb.2021.689466

Mauguen, A., Seshan, V. E., Ostrovnaya, I., and Begg, C. B. (2019). An EM Algorithm to Improve the Estimation of the Probability of Clonal Relatedness of Pairs of Tumors in Cancer Patients. BMC Bioinformatics 20, 555. doi:10.1186/s12859-019-3148-z

Ostrovnaya, I., Seshan, V. E., Olshen, A. B., and Begg, C. B. (2011). Clonality: an R Package for Testing Clonal Relatedness of Two Tumors from the Same Patient Based on Their Genomicprofiles. Bioinformatics 27, 1698-1699. doi:10.1093/ bioinformatics/btr267

Perea, J., García, J. L., Corchete, L., Lumbreras, E., Arriba, M., Rueda, D., et al. (2019). Redefining Synchronous Colorectal Cancers Based on Tumor Clonality. Int. J. Cancer 144, 1596-1608. doi:10.1002/ijc.31761 multiple primary tumors showing a clonal origin should be clearly differentiated from the others, maybe defined as multiple clonal neoplasms, and the heterogeneous multiple tumors occurring in the same individual may be the ones defined only as multiple primary tumors (synchronous or metachronous, using the concept of temporality, currently the only one used). We also keep in mind the possibility that clonal tumors could be a variety of metastatic disease, together with the diagnostic and therapeutic implications that this would involve.

\section{AUTHOR CONTRIBUTIONS}

All authors listed have made a substantial, direct, and intellectual contribution to the work and approved it for publication.

Schultheis, A. M., Ng, C. K. Y., De Filippo, M. R., Piscuoglio, S., Macedo, G. S., Gatius, S., et al. (2015). Massively Parallel Sequencing-Based Clonality Analysis of Synchronous Endometrioid Endometrial and Ovarian Carcinomas. JNCI.J 108 (6), djv427. doi:10.1093/jnci/djv427

Teixeira, M. R., Ribeiro, F. R., Torres, L., Pandis, N., Andersen, J. A., Lothe, R. A., et al. (2004). Assessment of Clonal Relationships in Ipsilateral and Bilateral Multiple Breast Carcinomas by Comparative Genomic Hybridisation and Hierarchical Clustering Analysis. Br. J. Cancer 91 (4), 775-782. doi:10.1038/ sj.bjc.6602021

Wang, X., Fang, H., Cheng, Y., Li, L., Sun, X., Fu, T., et al. (2018). The Molecular Landscape of Synchronous Colorectal Cancer Reveals Genetic Heterogeneity. Carcinogenesis 39, 708-718. doi:10.1093/carcin/bgy040

Conflict of Interest: The authors declare that the research was conducted in the absence of any commercial or financial relationships that could be construed as a potential conflict of interest.

Publisher's Note: All claims expressed in this article are solely those of the authors and do not necessarily represent those of their affiliated organizations, or those of the publisher, the editors, and the reviewers. Any product that may be evaluated in this article, or claim that may be made by its manufacturer, is not guaranteed or endorsed by the publisher.

Copyright (c) 2022 Perea, Corchete, García, Urioste and González-Sarmiento. This is an open-access article distributed under the terms of the Creative Commons Attribution License (CC BY). The use, distribution or reproduction in other forums is permitted, provided the original author(s) and the copyright owner(s) are credited and that the original publication in this journal is cited, in accordance with accepted academic practice. No use, distribution or reproduction is permitted which does not comply with these terms. 